\title{
Determination of large deformation and fracture behaviour of starch gels from conventional and wire cutting experiments
}

\author{
C. Gamonpilas ${ }^{1,2^{*}}$, M. N. Charalambides ${ }^{1}$, J. G. Williams ${ }^{1}$ \\ ${ }^{1}$ Imperial College London, Mechanical Engineering Department, London SW7 2AZ, \\ UK, ${ }^{2}$ Current address: National Metal and Materials Technology Center (MTEC), 114 \\ Paholyothin Road, Pathumthani 12120, Thailand \\ chaiwutg@mtec.or.th, +66 2564-6500 ext. 4447, +66 2564-6446
}

\begin{abstract}
Large deformation and fracture properties of two types of starch gels were investigated through uniaxial compression, single edge-notched bend (SENB) and wire cutting experiments. Tests were performed at various loading rates and for various starch/powder concentrations (\%w/w). It was found that starch gels exhibit rate independent stress-strain behaviour but show rate-dependent fracture behaviour, i.e. stress-strain curves at three loading rates are similar but fracture stress and fracture strain increase with increasing strain rate. This is rather unusual and interesting behaviour. SENB and wire cutting experiments also revealed ratedependent fracture behaviour and that the true fracture toughness $\left(G_{c}\right)$ values increase with loading/cutting speeds and starch powder concentration. In addition, the $G_{c}$ values from wire cutting and SENB tests were in reasonable agreement. The wire cutting process was also studied numerically using finite element techniques. A nonlinear elastic constitutive relationship based on Ogden was used to model the starch gels and a frictionless condition was assumed at the wire-starch gel contact interface. A fracture criterion based on maximum principal strain was assumed for the prediction of the steady state cutting force.
\end{abstract}

Keywords: Starch gels, wire cutting, fracture toughness, finite element analysis 


\section{INTRODUCTION}

Large deformation and fracture properties of foods are very important characteristics for food technologists. Manufacturing processes involving large deformation, fracture or separation of the material, e.g. cutting, slicing etc, are widely used in the food industry [1-4]. Fracture of food also occurs during mastication when the structure of food is broken down and the flavour and aroma are released [5]. Therefore, understanding how food deforms and fractures is a necessary first step towards optimising food processes and designing new consumer products. Large deformation behaviour of foods is commonly obtained via the uniaxial compression experiment [6] while fracture behaviour can be investigated by the single edgenotched bend (SENB) test [1]. However, the use of the SENB test for food can be difficult as most food exhibits a high degree of rate dependence, non-linearity and low stiffness. For these reasons, a simpler alternative test based on wire cutting, which involves pushing wires with various diameters into a specimen to a steady state cutting stage, has been proposed for measuring the fracture toughness of food [1,5]. A typical force-displacement trace for a wire cutting test is shown in Fig. 1, demonstrating the two phases of the process. In the first phase, the wire indents into the material. At some point during the indentation, the material starts to fracture and a steady state cutting phase proceeds. 


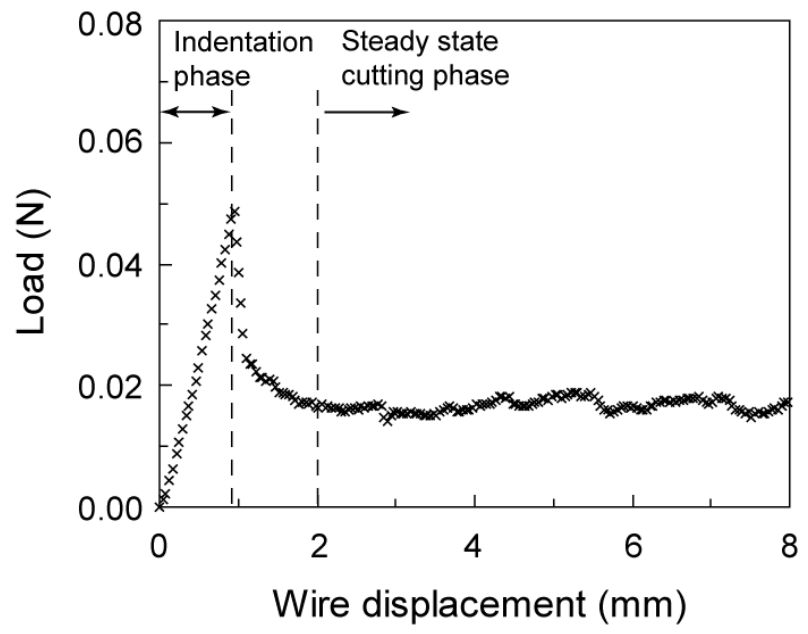

Fig. 1 Example of force-displacement relationship in the wire cutting test (2-34 (10\% $\mathrm{w} / \mathrm{w}$ ) gel, wire diameter of $0.25 \mathrm{~mm}$ and cutting speed of $5 \mathrm{~mm} / \mathrm{min}$ ).

In the wire cutting process, fracture, large deformation and surface friction occur simultaneously [1,5]. It has been stated by [7] that the force $F$ required for cutting is proportional to the wire diameter $(d)$ and that there is a constant component arising from $G_{c}$, the fracture toughness. The proportionality factor with the diameter was then determined from the deformation energy and the friction. A linear equation between the cutting force and wire diameter was developed by [1] and has the form,

$$
\frac{F}{b}=G_{c}+\left(1+\mu_{k}\right) \sigma_{y} d
$$

where $b$ is the width of the sample, $\sigma_{y}$ is a characteristic stress and $\mu_{k}$ is the kinetic coefficient of friction.

Starch is used functionally in various food products as a thickener, stabiliser or gelling agent, which greatly affects food structure [8,9]. A fundamental knowledge of the role of starch in large deformation and fracture properties is 
therefore essential for the development of the desired mechanical properties of a food system. The objective of this work is to investigate the large deformation and fracture behaviour of starch gels using conventional and wire cutting experiments. In addition, a finite element analysis of the wire cutting process to predict the steady state cutting force will be presented.

\section{EXPERIMENTS}

Two different types of starch gels, made from modified sago starch (here called material 2-34) and modified maize starch (here called material 2-12) were investigated. Gel samples were prepared as described in [10]. Prior to making the gel samples, the starch powders were dried overnight in an oven at $110{ }^{\circ} \mathrm{C}$ to remove any moisture present. From this point, the powders were kept in a desiccator and were only removed just before testing. Starch gels were made by mixing the powder with distilled water at various weight ratios (\% w/w) in a beaker. The beaker was then placed in a tub of boiling water. The mixing solution was stirred well for two minutes during which the gel reached its gelatinization state. The heating time was kept constant for every batch to ensure consistency and to avoid over cooking. Before transferring the solution into moulds, it was kept in the beaker at approximately $90{ }^{\circ} \mathrm{C}$ for a further 1 minute for the 2-34 gel and 18 minutes for the 212 gel. During this period, the beaker was sealed with a cling film to maintain the water content of the solution. Subsequently, the solution was poured into moulds and allowed to solidify over night, i.e. for approximately 15 hours, at room temperature. The gel settling time and temperature were kept approximately constant across batches to avoid any effects of storage and ageing. Uniaxial compression, wire 
cutting and SENB experiments were performed using the Instron 5543 machine at $21^{\circ} \mathrm{C}$ and $50 \%$ relative humidity. Two powder concentrations, e.g. $15 \% \mathrm{w} / \mathrm{w}$ and $20 \% \mathrm{w} / \mathrm{w}$, were used for each starch, although not all of these concentrations were used in the fracture tests.

\subsection{Uniaxial Compression tests}

Compression tests were performed on cylindrical samples with a diameter and height of $20 \mathrm{~mm}$. Three constant loading strain rates of $0.1,1$ and $10 / \mathrm{min}$ were used. Silicon oil was applied at the interface between sample and compression platens in order to minimise friction and to eliminate sample height effects [11]. A 100N load cell was used. At least six replicates were tested at each loading rate.

\subsection{Wire cutting tests}

Wire cutting tests, as shown in Fig. 2(a), were performed at three cutting speeds (V) of $0.5,5$ and $50 \mathrm{~mm} / \mathrm{min}$. Various powder concentrations were studied i.e. 15\% w/w and $20 \% \mathrm{w} / \mathrm{w}$ for $2-34$ and only $20 \% \mathrm{w} / \mathrm{w}$ for $2-12$. Steel wires of diameter, $d$, of 0.1 , $0.25,0.4$ and $0.5 \mathrm{~mm}$ were used. The specimens for the wire cutting tests were rectangular blocks of length $30 \mathrm{~mm}$, width $20 \mathrm{~mm}$ and height $20 \mathrm{~mm}$. A 10N load cell was employed since the cutting load on gels was generally small. For each case of $d$ and $V$, at least six replicates were tested. The steady-state cutting energy was obtained by dividing the cutting force, $F$, by the specimen width, $b$ (see Eq.1). 


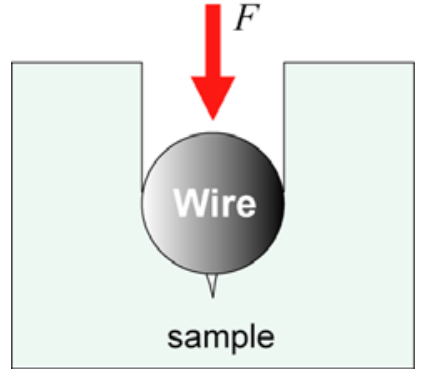

(a)



(b)

Fig. 2 Schematic representations of fracture tests (a) wire cutting and (b) SENB.

\subsection{Single edge-notched bend (SENB) tests}

Single edge-notched bend (SENB) tests, shown in Fig. 2(b), were conducted according to the ESIS protocol described in [12]. Specimens were rectangular blocks of length, $L=90 \mathrm{~mm}$, width, $w=20 \mathrm{~mm}$ and breadth, $b=10 \mathrm{~mm}$ (see Fig. 2(b)). A span, $S$, of $80 \mathrm{~mm}$ and a notch depth, $a$, of approximately $2 \mathrm{~mm}$ were used in these tests. The specimen notch size did not follow the ESIS protocol. This modification was necessary to prevent immediate fracture due to the self weight of the sample. The effect of notch length was not studied here but has been reported in [7]. Similar to the wire cutting experiments, tests were performed at three constant crosshead speeds of $0.5,5$ and $50 \mathrm{~mm} / \mathrm{min}$. At least four replicates were used for each speed. Only two materials, e.g. 2-34 (15\% w/w) and 2-34 (20\% w/w), were investigated as the others were too compliant and soft for this type of test. 


\section{EXPERIMENTAL RESULTS}

\subsection{Uniaxial compression tests}

Figs. 3(a) and 3(b) show the compression behaviour of 2-34 and 2-12 gels with 15\% $\mathrm{w} / \mathrm{w}$ and $20 \% \mathrm{w} / \mathrm{w}$ powder concentrations at three constant strain rates of $0.1,1$ and 10 /min, respectively. It was observed that samples did not barrel during compression tests due to the use of silicon oil. In addition, independent loading-unloading tests showed reversible response of the starch gels and samples always reverted back to their original shape upon removal of load although these results are not presented here. Both gels can therefore be assumed to deform in a non-linear elastic manner and are rate independent. This finding agrees with the previous work on starch gels [7,13]. In addition, these figures reveal that fracture stress and fracture strain increase with increasing deformation rate, suggesting that the fracture behaviour of starch gels is rate dependent.

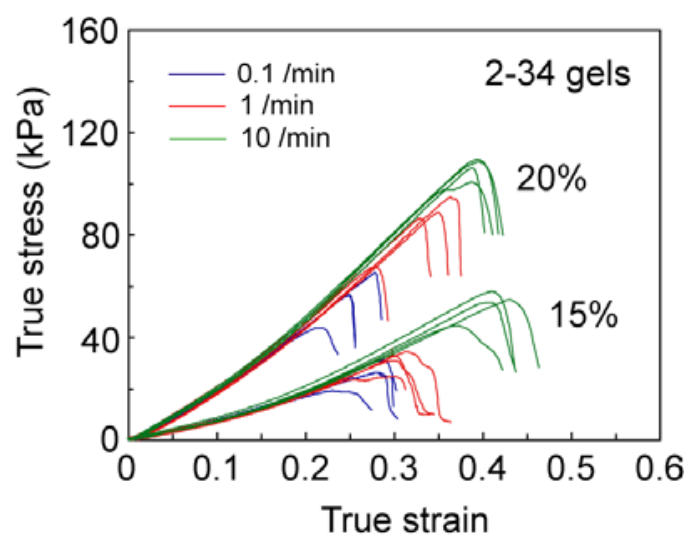

(a)

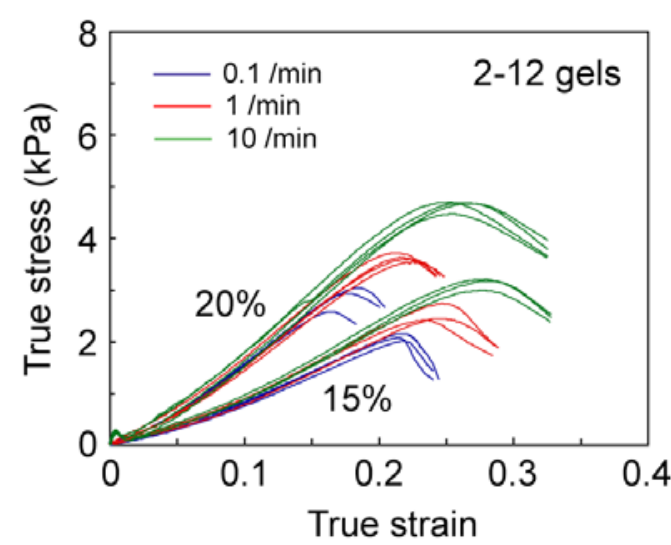

(b)

Fig. 3 Experimental measurements of compression response of (a) 2-34 and (b) 2-12 starch gels. 
The compression response of both 2-34 and 2-12 starch gels at various powder concentrations was calibrated with the Ogden model [14] in which the true stress, $\sigma$, under a uniaxial deformation state is defined as

$$
\sigma=\frac{2 \mu}{\alpha}\left(\lambda^{\alpha}-\lambda^{-\frac{1}{2} \alpha}\right)
$$

where $\lambda$ is the stretch ratio and $\lambda=\exp (\varepsilon)$ where $\varepsilon$ is the true strain, $\mu$ is the initial shear modulus and $\alpha$ is the Ogden constant. It is worth noting that at small strain Eq. (2) can be differentiated to give $\frac{\partial \sigma}{\partial \varepsilon}=E=3 \mu$. Using a Solver tool in Excel, the values of $\mu$ and $\alpha$ were obtained for each material. Fig. 4 compares the fittings from the model with the average experimental data corresponding to three loading rates up to the fracture points. The results show excellent agreement between the model and the experimental data, confirming the suitability of the model used. Furthermore, values of the material constants, $\mu$ and $\alpha$, for each material are tabulated in Table 1 . It is evident that starch gel 2-12 is a great deal less stiff than 2-34. 


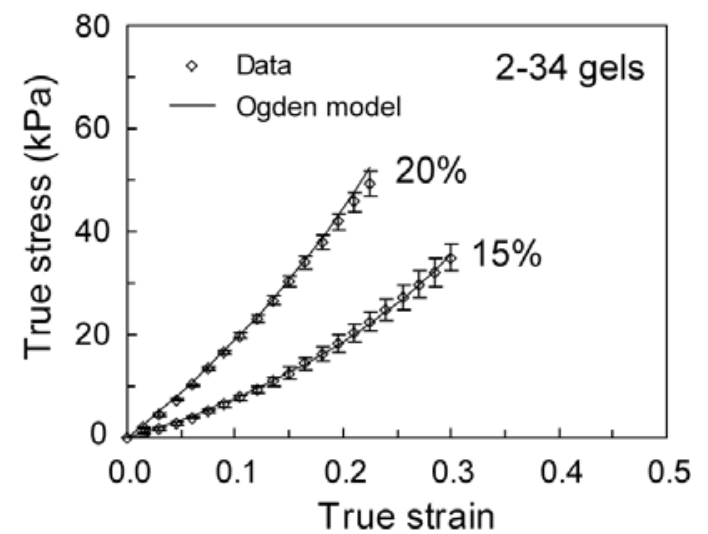

(a)



(b)

Fig. 4 Compression response of (a) 2-34 and (b) 2-12 at 15\% w/w and 20\% w/w fitted with the Ogden model described in Eq. (2).

Table 1 Calibrated material parameters of 2-34 and 2-12 gels at various powder concentrations.

\begin{tabular}{ccccc}
\hline Ogden & $2-34(15 \%)$ & $2-34(20 \%)$ & $2-12(15 \%)$ & $2-12(20 \%)$ \\
\hline$\mu(\mathrm{kPa})$ & 21.88 & 55.29 & 2.15 & 4.67 \\
$\alpha$ & -5.10 & -4.43 & -6.56 & -3.73 \\
\hline
\end{tabular}

The fracture stress and fracture strain values taken from the point of failure in the compression stress-strain curves are plotted against each other for all strain rates and powder concentrations in Fig. 5. For each concentration, the plot shows that the fracture strain increases with increasing strain rate. In addition, it decreases with increasing powder concentration for the same rate of deformation. Conversely, the fracture stress is found to increase with increasing concentration, and also increases with increasing deformation rate. From the material point of view, the rate-dependent fracture behaviour of starch gels is rather extraordinary. Previous work has suggested 
that this rate dependent fracture behaviour may arise from energy dissipation due to frictional processes occurring between the granules sliding against each other at large deformation [7]. This proposition is, however, questionable as similar fracture behaviour was also found on a gelatin which does not contain any particles [10]. The explanation of this remains a challenge as the large deformation and fracture behaviour of gels is related to their structure in a more subtle way than for small deformation behaviour. Different energy-dissipating mechanisms may occur; relationships are therefore not simple and depend on the structure of the gel [15]. In our recent work on gels to study the effect of temperature on deformation and fracture, it was observed that, conversely to the strain rate effect, both deformation and fracture depended on temperature [16].

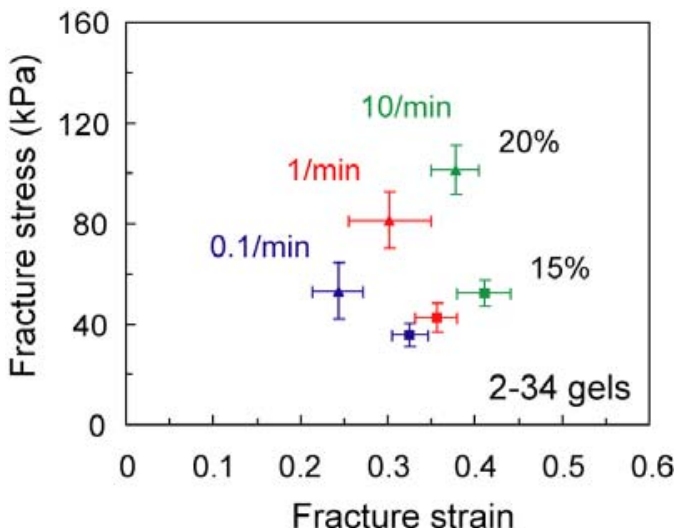

(a)



(b)

Fig. 5 Relationships between fracture stress and fracture strain of (a) 2-34 and (b) 212 starch gels at $15 \% \mathrm{w} / \mathrm{w}$ and $20 \% \mathrm{w} / \mathrm{w}$. 


\subsection{Wire cutting tests}

Fig. 6 shows the force-displacement curves from wire cutting tests on $2-34$ (15\% $\mathrm{w} / \mathrm{w}$ ) for wire diameters of $0.1,0.25,0.4$ and $0.5 \mathrm{~mm}$ and cutting speeds of $0.5,5$ and $50 \mathrm{~mm} / \mathrm{min}$. For each wire diameter, the indentation part of the curves is found to be similar although with different peak values for all cutting speeds, suggesting that the deformation properties are rate independent. The differences in the peak values are due to the fact that crack initiation depends on sample/material imperfections which vary from sample to sample. However, it was observed that the steady state force values increase with cutting speeds for all wire diameters. As a result, it can be concluded that the fracture behavior is rate dependent which is consistent with the compression results shown in the previous section. There is also a notable fluctuation, which seems to increase with the diameter of the cutting wire. This large scatter at larger diameters was also observed in the wire cutting study on cheese and it was attributed to secondary micro-cracking [1].



(a)

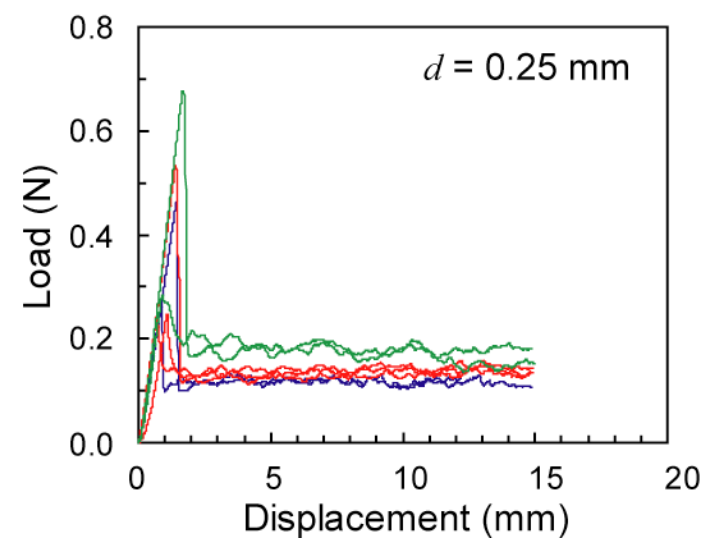

(b) 


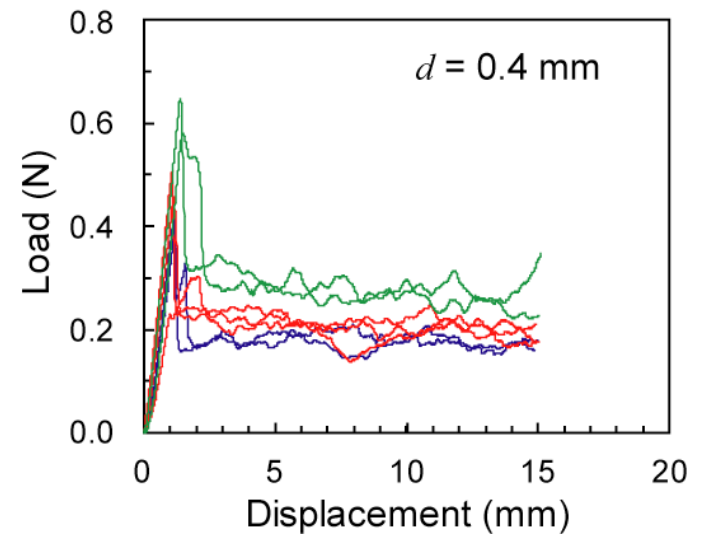

(c)

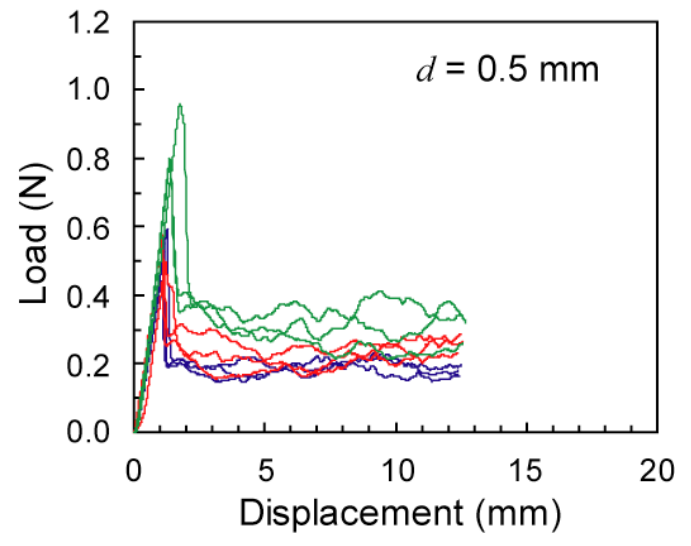

(d)

Fig. 6 Typical wire cutting data for 2-34 $(15 \% \mathrm{w} / \mathrm{w})$ at different cutting speeds and wire diameters of (a) 0.1, (b) 0.25, (c) 0.4 and (d) $0.5 \mathrm{~mm}$.

The normalised values of steady state cutting force $(F / b)$ were averaged and plotted against wire diameter as shown in Figs. 7(a)-(c) for 2-34 (15\% w/w), 2-34 $(20 \% \mathrm{w} / \mathrm{w})$ and $2-12(20 \% \mathrm{w} / \mathrm{w})$, respectively. It can be seen that the steady state cutting force increases almost linearly with wire diameter for all materials. The relationship was fitted with Eq. (1) to obtain the fracture toughness, $G_{c}$, and the characteristic stress and friction coefficient, $\sigma_{y}\left(1+\mu_{k}\right)$. The fracture toughness was assumed to be constant for different wire diameters and was extrapolated to the theoretical zero wire diameter. The fits are generally good with $R^{2}$ greater than 0.95 for all cases. The values of $G_{c}$ were found to be rate dependent and increase with increasing cutting speeds. In addition, the values are relatively small as expected and are found to be between $1.9-3.1 \mathrm{~J} / \mathrm{m}^{2}$ for the 2-34 gels and $0.3-1.2 \mathrm{~J} / \mathrm{m}^{2}$ for the $2-12$ gel. For comparison purposes, the values of $G_{c}$ for a $10 \% \mathrm{w} / \mathrm{w}$ potato starch gel are between 0.7-10 J/m $\mathrm{m}^{2}$ for cutting speeds between $0.2-500 \mathrm{~mm} / \mathrm{min}$ [7] and for mild 
Cheddar and Gruyere are between 14.7-33.6 $\mathrm{J} / \mathrm{m}^{2}$ for cutting speeds between 5-500 $\mathrm{mm} / \mathrm{min}[4]$.

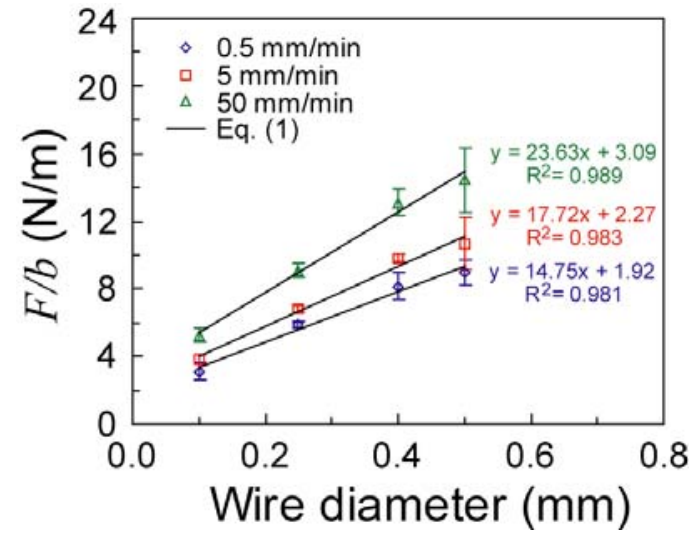

(a)



(b)

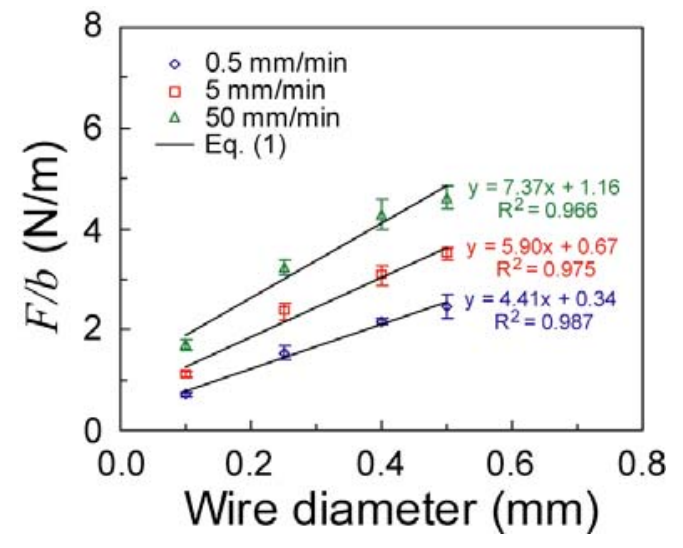

(c)

Fig. 7 Experimental wire cutting data at different cutting speeds for (a) 2-34 (15\% w/w), (b) 2-34 (20\% w/w) and (c) 2-12 (20\% w/w).

\subsection{Single edge-notched bend tests}

Figs. 8(a) and 8(b) show load-displacement curves at three loading speeds for the

SENB tests on 2-34 (15\% w/w) and 2-34 (20\% w/w) gels, respectively. The loaddisplacement data shown in Fig. 8 were used to calculate the fracture toughness and 
Young's modulus as recommended in the ESIS protocol [12]. Due to sagging of the samples due to self weight (see Fig. 9), the data shown in Fig. 8 had to be corrected prior to the calculations of $G_{c}$ and $E$. This was done by adding an equivalent central load, $\bar{F}$, which gives the same bending moment at the middle of the SENB specimen due to its own weight. Appendix A describes how this correction is computed. The equivalent load was found to be equal to

$$
\bar{F}=N\left(1-\frac{L}{2 S}\right)
$$

where $N$ is the specimen weight. For these SENB specimens $(L=90 \mathrm{~mm}$ and $S=80$ $\mathrm{mm}$ ), the equivalent load of $7 / 16$ of the specimen weight was added to the measured loads.

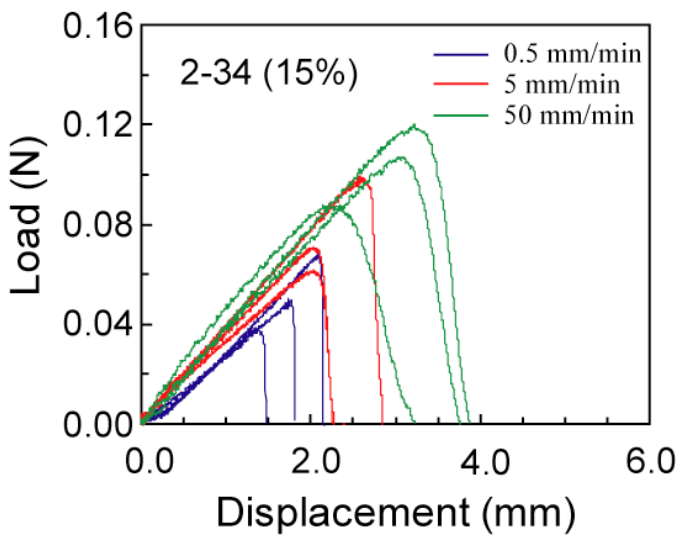

(a)

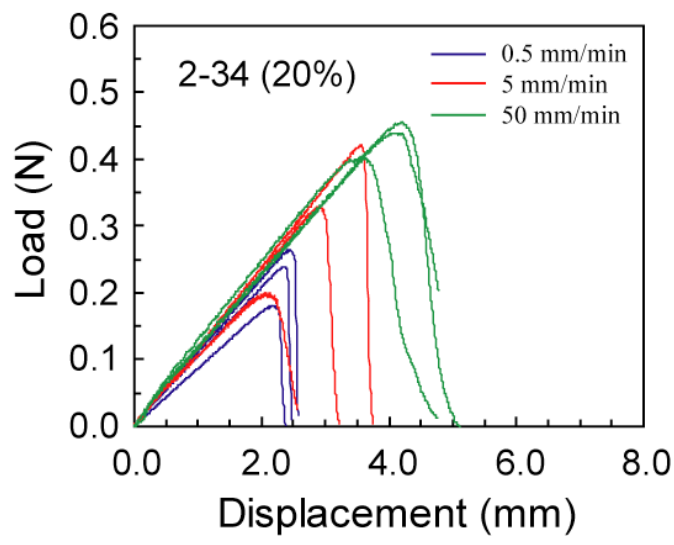

(b)

Fig. 8 Experimental SENB data at different crosshead speeds for (a) 2-34 (15\%) and (b) 2-34 (20\%). 




Fig. 9 Sagging of SENB sample prior to loading.

The calculations of $G_{c}$ and $E$ from the corrected SENB results pose the further question as to what are the corresponding crack speeds. In the SENB tests, two types of behaviour can be observed. For large crack lengths, the cracks grow in a stable manner and the crack decelerates during propagation. It is thus possible to calculate the initial, i.e. initiation, speed which may be ascribed to the initiation $G_{c}$ value. For small crack lengths, the initiation is unstable but then arrests at a larger value of crack length but at the same $G$ and propagates in a stable manner thereafter. This initiation value of crack length after arrest can be used with the initiation $G_{c}$ value to calculate the crack speed. The calculation of crack speed is described in Appendix B. The crack speed is given by $(X w) / t$ where $X$ depends on the ratio between notch depth and specimen width $(a / w)$ and $t$ is the fracture initiation time. In the SENB tests reported here, $a / w=0.1$ and $X$ is equal to 0.641 . The crack speeds are given in Table 2 .

For comparison purposes, the $G_{c}$ and $\sigma_{y}\left(1+\mu_{k}\right)$ values of $2-34(15 \% \mathrm{w} / \mathrm{w}), 2-$ $34(20 \% \mathrm{w} / \mathrm{w})$ and $2-12(20 \% \mathrm{w} / \mathrm{w})$ from the wire cutting experiments are also presented in Table 2. Additionally, comparisons of the $G_{c}$ values from the two tests 
are shown graphically in Figs. 10(a) and 10(b) for 2-34 (15\%) and 2-34 (20\%) as $G_{c}$ versus crack or wire cutting speeds. The standard deviations of the $G_{c}$ from the wire cutting data were calculated using a linear regression analysis described in [17]. It is seen that the $G_{c}$ values predicted from both tests are generally in good agreement. The data can be represented in a power law form,

$$
G_{c}=G_{0} \dot{a}^{n}
$$

where $G_{0}$ is the value at $\dot{a}$ (crack speed) $=1 \mathrm{~mm} / \mathrm{min}$. For the $2-34$ gels, $G_{0}=1.9$ $\mathrm{J} / \mathrm{m}^{2}$ and $n=0.09$ for $15 \% \mathrm{w} / \mathrm{w}$ and $G_{0}=2.3 \mathrm{~J} / \mathrm{m}^{2}$ and $n=0.14$ for $20 \% \mathrm{w} / \mathrm{w}$ (see Fig. 10). Only wire cutting data is available for fitting the $2-12(20 \% \mathrm{w} / \mathrm{w})$ material. The fit gives $G_{0}=0.4 \mathrm{~J} / \mathrm{m}^{2}$ and $n=0.27$.

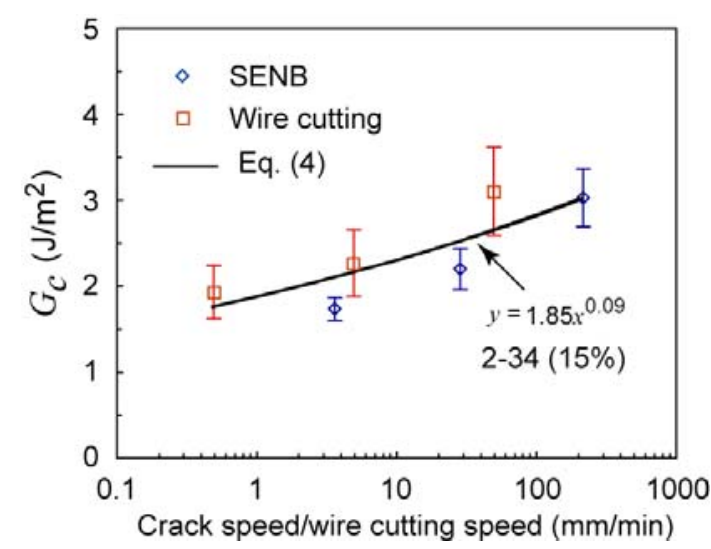

(a)

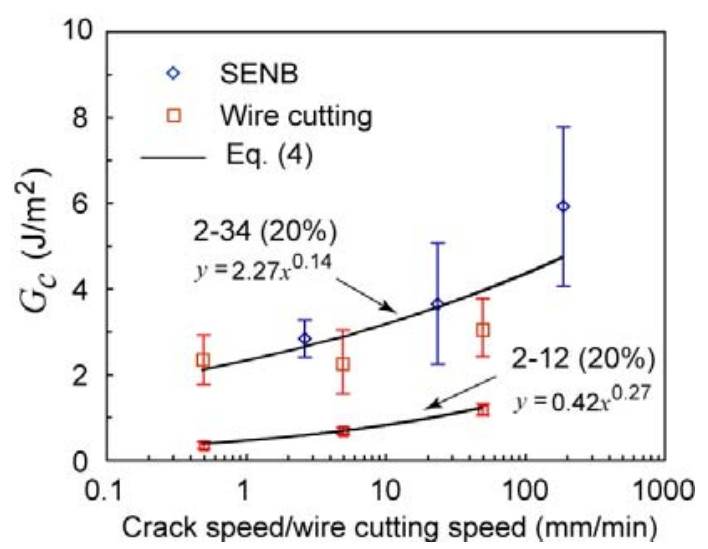

(b)

Fig. 10 Comparison of fracture toughness values predicted from SENB and wire cutting tests for (a) 2-34 (15\% w/w) and (b) 2-34 (20\% w/w) and 2-12 (20\% w/w).

The calculations of Young's moduli of 2-34 (15\% w/w) and 2-34 (20\% w/w) from the SENB data are also shown in Table 2. It can be seen that the Young's moduli for both materials do not change significantly with the SENB crosshead 
speeds. The average values were found to be $63.7 \pm 11.8 \mathrm{kPa}$ and $162.3 \pm 29.1 \mathrm{kPa}$ for 2-34 (15\% w/w) and 2-34 (20\% w/w), respectively. In addition, the Young's moduli of the 2-34 $(15 \% \mathrm{w} / \mathrm{w})$ and $2-34(20 \% \mathrm{w} / \mathrm{w})$ gels, calculated from the compression data shown in Table 1 as $3 \mu$, are 65.6 $\pm 3.2 \mathrm{kPa}$ and $165.9 \pm 4.6 \mathrm{kPa}$, respectively. It is apparent here that the agreement between the two tests is very good, confirming the rate-independence of the stress/strain behaviour. 
Table 2 Summary of $G_{c}$ values calculated from wire cutting and SENB experiments.

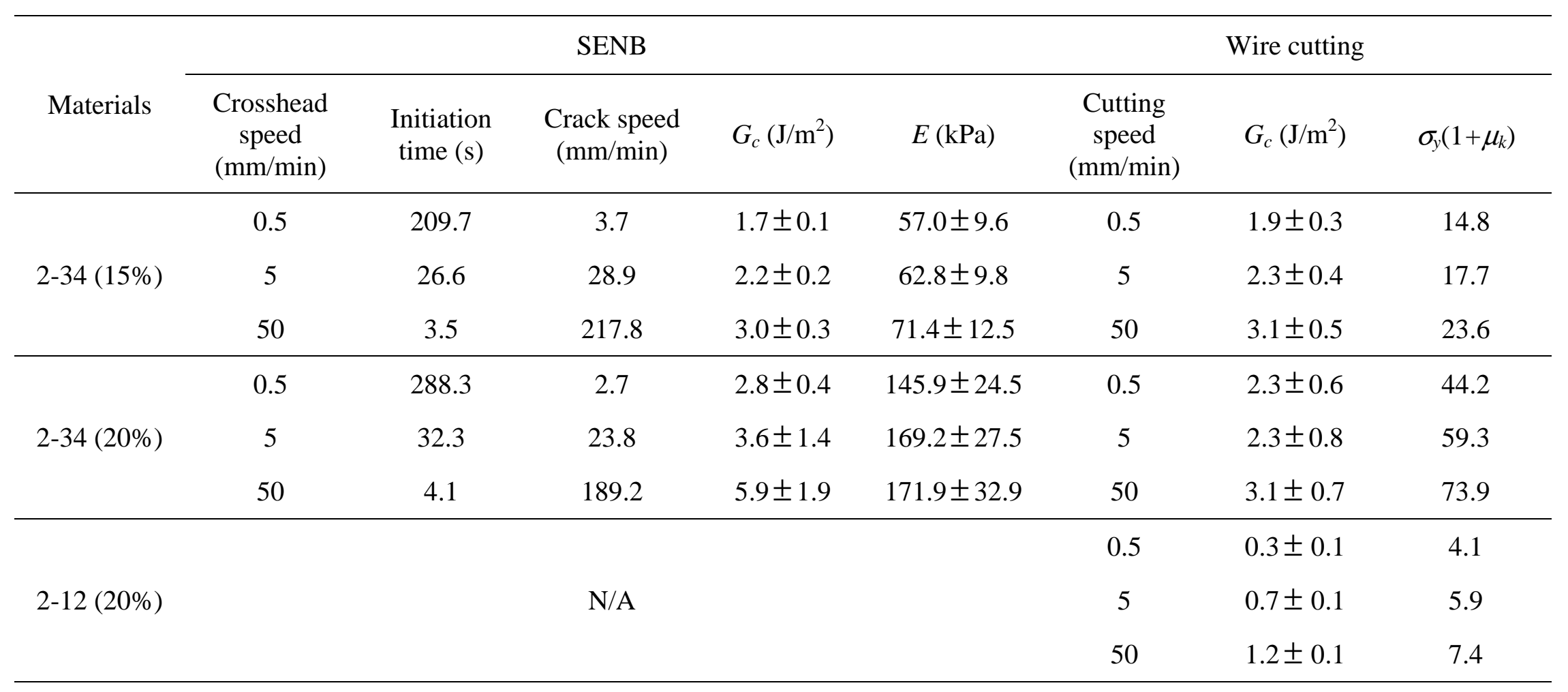




\section{Numerical simulation of wire cutting process}

Finite element simulations were performed to study the wire cutting process and to predict the steady state cutting force at various wire diameters. The simulations were performed using the commercial finite element code ABAQUS [18]. The geometry of the FE model is based on the actual specimen sizes and is shown schematically in Fig. 11(a). The bulk material was modelled using four noded, plane strain solid elements with the material parameters taken from those calibrated from the monotonic compression data. A hyperelastic model based on Ogden was chosen as the constitutive model (see Eq. (2)). Simulations were only performed for the 2-34 (15\% w/w) gel. The wires were defined as rigid surfaces and were prescribed to move along the $y$-direction. The bottom surface of the model was constrained in the $y$-direction but was allowed to move freely in the $x$ direction. A finer mesh was used along the cutting path to improve the accuracy and convergence of the solution (Fig. 11(b)). An initial notch shown in Fig. 11 is needed to help with the numerical convergence of the crack propagation simulation. In addition, the simulation is concerned with the prediction of steady state forces so the initial notch is not important.

The steady state cutting phase was simulated using a node-release technique. This was implemented through the MPC subroutine in ABAQUS [18]. It was assumed that the crack grows in the plane of cutting, i.e. along the symmetry plane. The nodes along the cutting path are released, and the crack

allowed to propagate, when the maximum principal strain at a distance $l$ ahead of the wire attains a critical value (see Fig. 11(b)). The FE model of the indentation phase, i.e. without crack node release, was used to determine the distance $l$ which was taken to be equal to the distance from the initial crack tip (bottom of the wire) 
to the point where the principal strain attained its maximum value. This can be demonstrated by the contour plot of the maximum principal strain in the region around the cutting in Fig. 12. It is noted that the critical distance changes with the size of wire diameter and does not vary significantly with the wire displacement. Values of the distance $l$ are approximately equal to $0.06,0.09$ and $0.12 \mathrm{~mm}$ for the wire diameters of $0.25,0.4$ and $0.5 \mathrm{~mm}$, respectively.

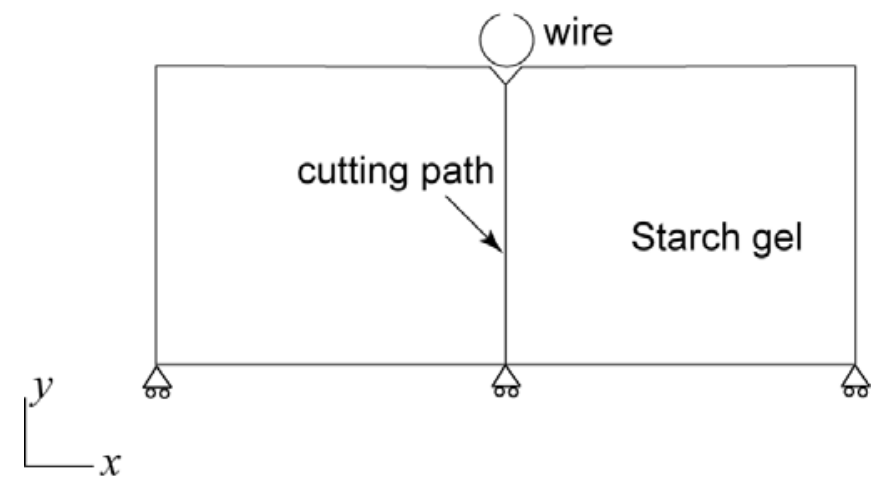

(a)

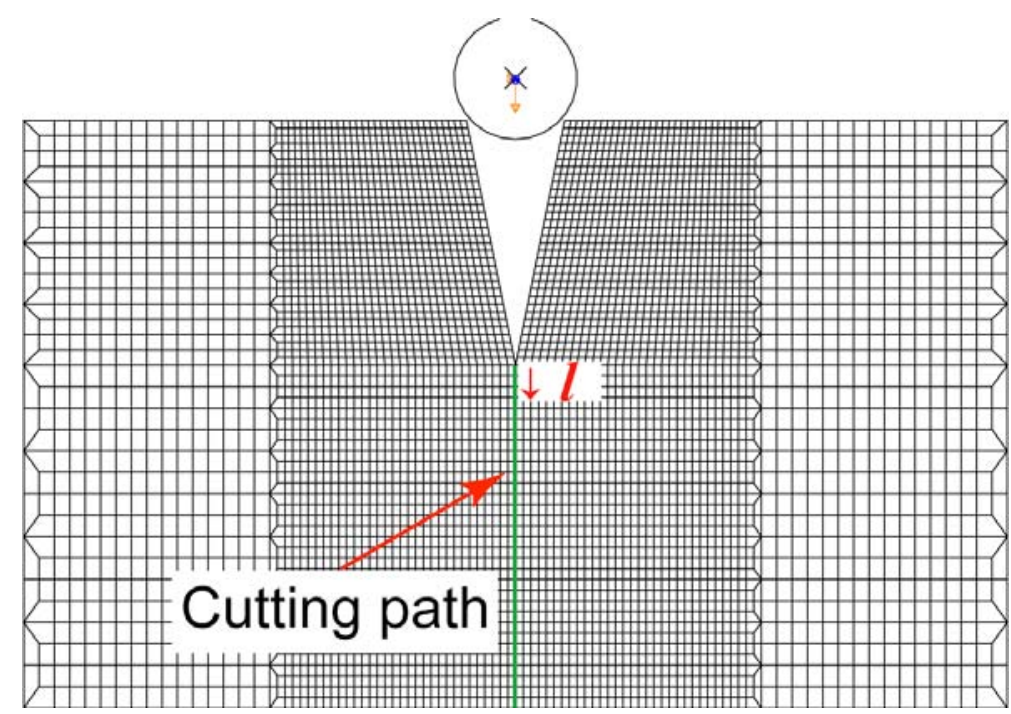

(b)

Fig. 11 (a) Finite element geometry for wire cutting model and (b) typical mesh used in simulation. 


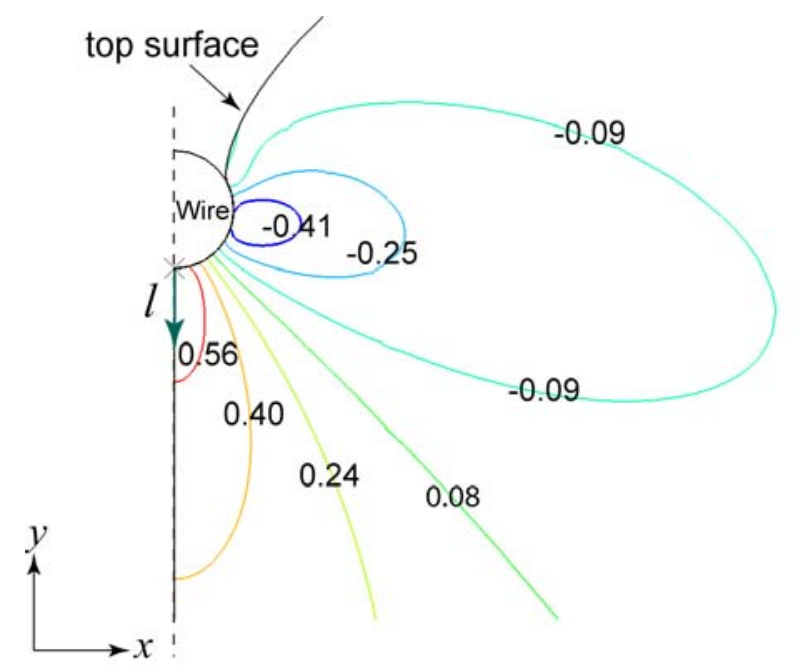

Fig. 12 Contour plot of the maximum principal strain for the wire cutting simulation on 2-34 $(15 \% \mathrm{w} / \mathrm{w})$ gel with $d=0.4 \mathrm{~mm}$, demonstrating the critical distance $l$ where maximum strain occurs.

The critical strain value as a function of cutting speed was first determined such that the cutting force predictions matched the experimental data for the wire diameter of $0.25 \mathrm{~mm}$ (see Fig. 13(a)). The initial 'slack' in the FE simulations is due to the presence of the notch needed in FE the model. All in all, the steady state cutting forces from the FE simulations are in good agreement with the wire cutting experiments at all cutting speeds. These critical strain values were found to be $0.15,0.17$ and 0.22 for the cutting speeds of $0.5,5$ and $50 \mathrm{~mm} / \mathrm{min}$, respectively. In order to verify these critical strain values, simulations were performed on the bigger wire diameters of 0.4 and 0.5 . For these diameters, the same critical strain values were used as for the $0.25 \mathrm{~mm}$ diameter case, whereas the critical distance $l$ was determined from the models of the indentation phase as described previously. The predicted steady state values of $F / b$ are shown in comparison with the experimental data in Fig. 13(b). Reasonable agreement between the prediction of the steady-state cutting force and the experimental data is obtained for the diameter of $0.4 \mathrm{~mm}$ at all speeds whereas the error for the 0.5 mm diameter wire reaches a maximum of $20 \%$ at the speed of $50 \mathrm{~mm} / \mathrm{min}$. 


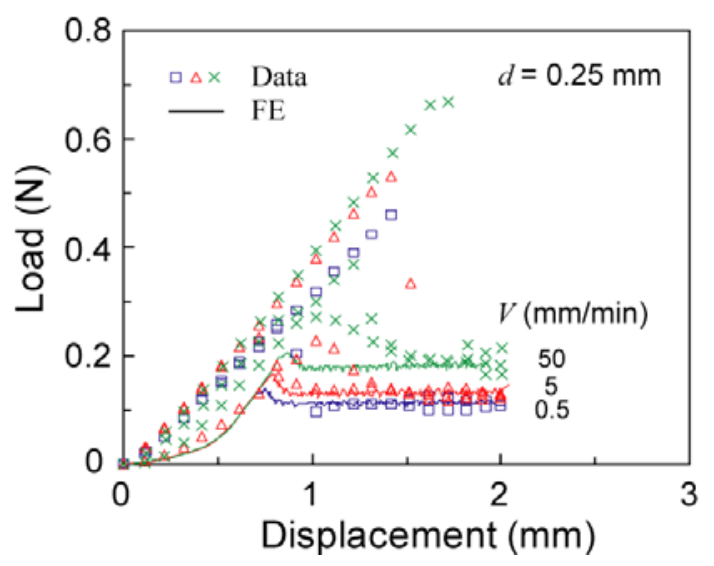

(a)

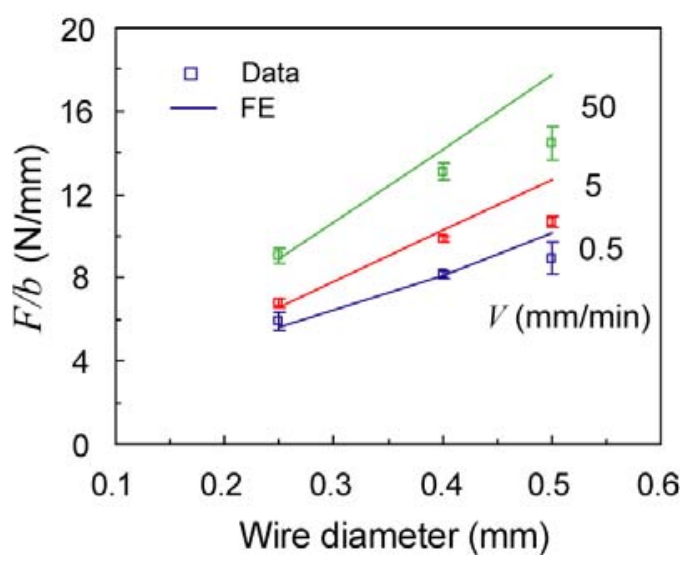

(b)

Fig. 13 Comparison of steady state cutting forces $(F / b)$ between FE simulations and experimental data for 2-34 (15\% w/w) with (a) $d=0.25 \mathrm{~mm}$ and (b) various wire diameters.

The critical strain values at each cutting speed are compared with the fracture strains from the compression data in Fig. 14. Wire cutting speeds was converted into their corresponding strain rates through the chain rule, $\frac{d \varepsilon}{d t}=\frac{d \varepsilon}{d \delta} \times \frac{d \delta}{d t}$ where $\delta$ is the displacement and $\frac{d \delta}{d t}$ is the cutting speed. The expression $\frac{\partial \varepsilon}{\partial \delta}$, which describes how the maximum tensile strain changes with the wire displacement, was obtained from the FE models of the indentation phase. The comparison shown in Fig. 14 is encouraging as both strains follow the same trend with increasing rate. In addition, the critical strain values found from the wire cutting tests, which can be assumed to represent the tensile fracture strains, are approximately one half of the compressive fracture strains. This ratio is interestingly coincident with the previous work on potato starch gels which found that fracture strains under uniaxial compression at three loading rates were approximately twice the values of those obtained under uniaxial tension tests [19]. 
These results should be further investigated and could be verified by performing uniaxial tension experiments.



Fig. 14 Comparison of critical strain and fracture strain values of 2-34 (15\% w/w) from wire cutting simulations and compression data.

\section{CONCLUSION}

The deformation response of starch gels shows rate independent, non linear elastic behaviour that can be described by the Ogden constitutive model. However, the fracture behaviour is rate dependent. In particular, both fracture stress and fracture strain from uniaxial compression tests increase with increasing deformation rates and powder concentrations $(\% \mathrm{w} / \mathrm{w})$. The values of fracture toughness obtained from wire cutting experiments were found to be relatively small and were consistently rate dependent. In addition, these values were in reasonable agreement with those obtained from SENB tests, confirming the reliability of the cutting experiment. The numerical simulation of the wire cutting process, assuming the calibrated Ogden model and a critical maximum strain criterion, was reasonably successful in predicting the steady state cutting forces at various cutting speeds and wire diameters. Further work is required to verify this 
technique by using it to predict failure during wire cutting of all the different gels presented here as well as during the SENB tests. Alternative failure criteria such as the Cohesive Zone model [5] should also be investigated; the latter would use a traction-separation law - with energy release rate being the area under this traction-separation curve - to describe the behaviour of special elements placed along the wire path. Regarding the rate dependent fracture properties, a study is currently underway where compression and cutting tests are being performed at different temperatures. This might lead to a better understanding of the rather peculiar behaviour of these gels, i.e. the rate dependence of fracture in contrast to the rate independence of deformation properties.

\section{Acknowledgements}

Support for this work has been provided by ICI under the SRF scheme. The authors would like to thank Drs Dooling and Gibbon of ICI (Akzo Nobel) for their advice and useful discussions. ABAQUS was provided under academic license by HKS Inc., Providence, Rhode Island, USA. 


\section{APPENDIX A: Self Weight Correction}

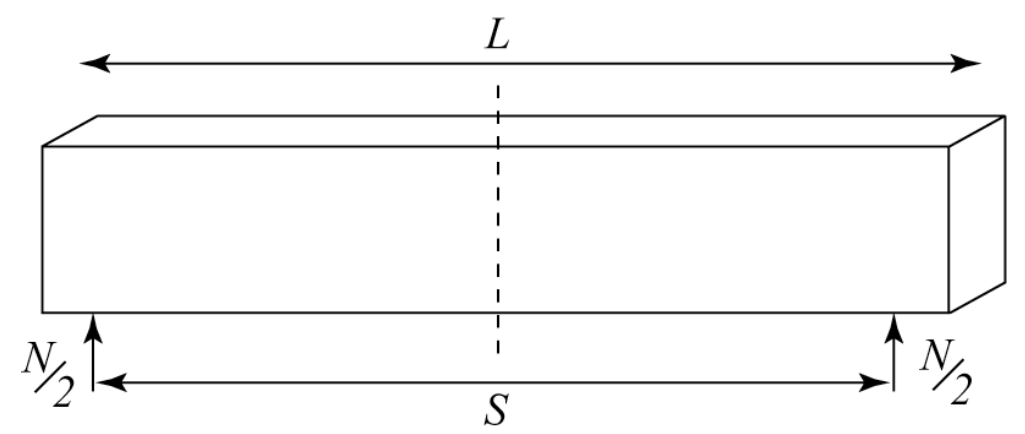

Let weight of beam be $N$. Moment at the centre of the beam due to $N$ is

$$
M=\left(\frac{N}{2}\right)\left(\frac{S}{2}\right)-\left(\frac{N}{2}\right)\left(\frac{L}{4}\right)=\frac{N S}{4}\left(1-\frac{L}{2 S}\right)
$$

This is equivalent to a central force $\bar{F}$;

$$
M=\frac{\bar{F} S}{4}
$$

and hence

$$
\bar{F}=N\left(1-\frac{L}{2 S}\right)
$$

In these specimens $L=90 \mathrm{~mm}$ and $S=80 \mathrm{~mm}$, therefore

$$
\frac{\bar{F}}{N}=\frac{7}{16}
$$

The total moment is obtained by adding $\bar{F}$ to $F$, the measured load. Note that if $L=2 S$, there is no correction. 


\section{APPENDIX B: Initiation Crack Speeds}

Tests using cutting are run at fixed speeds and the toughness is generally assumed to be a function of this speed which is equivalent to a crack speed. Comparative tests would be best performed on stable, fixed crack speed, tests such as the tapered DCB or Double Torsion. However, such tests are difficult for soft solids, and single edge-notched bend (SENB) tests have been used as in this work. In such tests two types of behaviour are observed. For large crack lengths the cracks grow in a stable manner and the crack decelerates during propagation. It is thus possible to calculate the initial, i.e. initiation, speed which may be ascribed to the initiation $G_{c}$ value. For low crack lengths the initiation is unstable but then arrests at a larger value and propagates in a stable manner thereafter. This initiation value after arrest can be used with the initiation $G_{c}$ value.

The crack speeds may be determined as follows. For an SENB specimen loaded at a constant speed, $V, G$ is given by;

$$
G=\frac{P^{2}}{2 b} \frac{d C}{d a}
$$

where $P$ is the load, $b$ is the specimen thickness, $a$ is the crack length and $C$ is the specimen compliance,

$$
C=\frac{V t}{P}
$$

and $t$ is the time.

Substituting for $P$ we have, 


$$
\left(\frac{2 b w G}{V^{2}}\right)=\frac{t^{2}}{C^{2}} \frac{d C}{d x}=\frac{t^{2}}{C \phi}
$$

where $t$ is the fracture time, $w$ is the specimen width and $x=a / w$. Now the energy calibration factor $\phi$ is given by [20],

$$
\phi=\frac{C}{d C / d x}
$$

If we assume that $G=G_{c}$ and is constant during propagation then,

$$
\begin{aligned}
& \text { i.e. } \frac{d}{d x}\left(\frac{2 b w G_{c}}{V^{2}}\right)=\frac{t}{C \phi}\left(\frac{2}{\dot{x}}-\frac{t}{\phi}\left(1+\frac{d \phi}{d x}\right)\right)=0 \\
& \dot{x}=\frac{\dot{a}}{w}=\frac{X}{t}, \quad X=\frac{2 \phi}{1+\frac{d \phi}{d x}}
\end{aligned}
$$

$\phi$ may be modelled as,

$$
\phi=\phi_{1} x^{-m}
$$

where $\phi_{1}$ and $m$ are constants and for the standard specimens with a span to depth ratio of $4, \phi_{1}=0.142$ and $m=0.787$ [20]. Thus we have,

$$
X=\frac{2 \phi_{1} x}{x^{1+m}-m \phi_{1}}
$$

For $x>\left(m \phi_{1}\right)^{\frac{1}{1+m}}$, then $X>0$ and we have stable crack growth initiating at a speed of $\dot{X}_{0}=\frac{X_{0}}{t_{0}}$ where $t_{0}$ is the initiation time and $X_{0}$ is $X$ evaluated at the initial value of $x=x_{0}$. For $x<\left(m \phi_{1}\right)^{\frac{1}{1+m}}$, then $\dot{x}<0$ so there is no real solution and the 
crack jumps forward to an $x$ value with the same $G$ value i.e. from $x_{0}$ to $x_{1}$. From B2 this condition is,

$$
C_{0} \phi_{0}=C_{1} \phi_{1}
$$

From the definition of $\phi$ we have

$$
\ln \left(\frac{C}{C_{0}}\right)=\int_{x_{0}}^{x_{1}} \frac{d x}{\phi}=-\ln \left(\frac{\phi_{1}}{\phi_{0}}\right)=0
$$

and hence

$$
x_{0}^{1+m}=\phi_{1} m\left[\frac{\ln \left(\frac{x_{1}}{x_{0}}\right)^{1+m}}{\left(\frac{x_{1}}{x_{0}}\right)^{1+m}-1}\right]
$$

from which pairs of $x_{0}$ and $x_{1}$ may be calculated. The speed at growth at $x_{1}$ may be computed from $X_{1}$.

The same result may be derived via B4 since this may be integrated to give,

$$
\ln \left(\frac{t}{t_{0}}\right)^{2}=\int_{x_{0}}^{x_{1}} \frac{d x}{\phi}+\ln \left(\frac{\phi_{1}}{\phi_{0}}\right)=0
$$

for $t=t_{0}$. A table of values is given below. 
Table B1: Calculations of the initial $a / w$ ratio, $x_{0}$, the arrest and new initiation ratio, $x_{1}$, and $X$ from B7 and B4 given that $\phi_{1}=0.142$ and $m=0.787$

\begin{tabular}{ccc}
\hline$x_{0}$ & $x_{1}$ & $X$ \\
\hline 0.052 & 0.69 & 0.49 \\
0.10 & 0.57 & 0.64 \\
0.14 & 0.49 & 0.83 \\
0.19 & 0.41 & 1.28 \\
0.24 & 0.35 & 2.41 \\
0.30 & 0.30 & $\infty$ \\
\hline
\end{tabular}

A similar analysis to this is given in [21], Chapter 3. In that case the crack speed is related to the testing rate $V$ and not $t$ as here but the method is the same. There is also discussion of the arrest condition in Chapter 8.

\section{References}

[1] Kamyab I, Chakrabarti S, Williams JG (1998) J Mater Sci 33:2763-70.

[2] Dobraszczyk BJ, Atkins AG, Jeronimidis G, Purslow PP (1987) Meat Sci 21:25-49.

[3] Xiao W, Charalambides MN, Williams JG (2006) Int J Food Sci Technol 42:699-707.

[4] Vincent JFV, Jeronimidis G., Khan AA, Luyten H (1991) J Texture Stud 22:47-55.

[5] Goh SM, Charalambides M, Williams JG (2005) Eng Fract Mech 72:931-946.

[6] Goh SM, Charalambides MN, Williams JG (2004) Mech Time-Depend Mater 8:255-268.

[7] Luyten H, Van Vliet T (1995) J Texture Stud 26:281-298.

[8] Galliard T (1987) Starch: Properties and potential, John Wiley \& Sons, UK. 
[9] Imeson A (1997) Thickening and gelling agents for food, Aspen publishers Inc, Maryland.

[10] Gamonpilas C, Charalambides MN, Williams JG, Dooling PJ, Gibbon SR (2009) submitted for publication.

[11] Charalambides MN, Goh SM, Wanigasooriya L, Williams JG, Xiao W (2005) J Mater Sci 40:3375-3381.

[12] Williams JG, Cawood MJ (1990) Polym Testing 9:15.

[13] Ikeda S, Sangu T, Nishinari K (2003) Food Sci Technol Res 9:372-377.

[14] Treloar LRG (1975) The physics of rubber elasticity, Oxford University Press, UK.

[15] van Vliet T, Walstra P (1995) Faraday Discuss 101:359-370.

[16] Charalambides MN, Gamonpilas C, Williams JG (2009) to be published.

[17] Williams JG, Rink M (2007) Eng Fract Mech 74:1009-1017.

[18] ABAQUS Version 6.4 (2004) Hibbitt Karlsson and Sorensen Inc, Providence, $R I$.

[19] Luyten H, van Vliet T, Walstra P (1992) J Texture Stud 23:245-266.

[20] Williams JG (1984) Fracture mechanics of polymers, Ellis Horwood Limited, UK.

[21] Atkins AG, Mai YW (1985) Elastic and plastic fracture, Ellis Horwood Limited, UK. 